Aus dem pathologischen Institut der Königl. Charité zu Berlin.

\title{
Ein Fall von zweieiiger Zwillingsschwangerschaft (vielleicht Superfoetatio).
}

\author{
Von
}

\author{
Dr. Bruno Wolff II. \\ (Mit 3 Abbildungen auf Tafel XI.)
}

In den geburtshülflichen Werken werden bei der Erörterung der Frage der Superfoetatio und Superfoecundatio im Allgemeinen die theoretischen Bedenken gegen die Möglichkeit der Superfoetatio erwähnt, und es wird betont, dass auch in der Praxis kein sicherer Beweis für das Vorkommen derselben bestehe.

In der Literatur erscheinen dann und wann Veröffentlichungen, in denen Fälle von sogenannter Superfoetatio beschrieben werden. Der Beweis für das Bestehen einer solchen wird meist durch Verschiedenartigkeiten in der Bildung der Zwillinge oder Drillinge geführt. Sie sind verschieden weit in der Entwicklung vorgeschritten. Dies wird aber, wie fast allgemein anerkannt wird, besser dadurch erklärt, dass durch die Raumbeengung im Uterus Störungen in der Entwicklung vorkommen, die ein sehr ungleiches Gewicht und auch anderweitige ungleiche Beschaffenheit zur Folge haben. Ferner werden als Beispiele für die Superfoetatio Fälle angegeben, in denen Zwillingsfrüchte in mehr oder weniger auseinander liegenden Zeiträumen geboren werden. Hierfür giebt Schröder die einfache Erklärung, „dass, nachdem die eine gut entwickelte Zwillingsfrucht geboren, die andere in der Entwicklung zurückgebliebene einstweilen zurückgehalten und erst nach Monaten, nachdem sie gehörig ausgebildet, ihrerseits ausgestossen würde." Andere Beobachtungen, besonders die Geburt eines weissen und 
schwarzen Kindes bei erfolgtem geschlechtlichen Verkehr der Mutter mit einem Neger und einem Weissen lassen sich besser als Superfoecundatio deuten, das heisst die Befruchtung mehrerer von derselben Ovulationsperiode herrührender Eier hat durch verschiedene Begattungsacte stattgefunden.

Jetzt neuerdings, seitdem man Ovulation und Menstruation nicht mehr für unbedingt von einander abhängig und nicht für stets gleichzeitig hält, ist, wie Hofmeier in seiner neuesten Ansgabe des Schröder'schen Handbuches sagt, der Unterschied zwischen Superfoecundatio und Superfoetatio nicht -mehr aufrecht zu erhalten, und es kommt nur darauf an zu beobachten, wie lange nach dem Eintritt der Conception noch eine weitere Befruchtung möglich ist.

Im Folgenden will ich einen Fall einer kurzen Besprechung unterziehen, bei dem ich den Nachweis führen will, dass längere Zeit nach dem Bestehen einer Schwangerschaft noch eine zweite Befruchtung stattfand. Wenn ich ihn unter dem Namen der Superfoetatio veröffentliche, so thue ich es, weil ich für denselben keinen anderweitigen, kurzen und prägnanten Ersatz weiss.

Am 6. October 1899 starb das 21 jährige Dienstmädchen I. K. Sie wurde am 7. October durch Herrn Professor Israel secirt, dem ich die Ueberlassung des Präparates verdanke.

Die Todesursache ist eitrige Peritonitis und allgemeine Sepsis, die von einem wahrscheinlich mit einer Nadel vorgenommenen missglückten Abtreibungsversuch herrühren. Es findet sich in der Cervix ein kleines Loch, durch welches die eingeführte Sonde in die Bauchhöhle gelangt. Um die Ausgangsöffnung des sondirten Ganges befinden sich eitrige Auflagerungen, die sich auch auf dem ganzen Peritoneum zeigen.

Der Uterus entspricht in seiner Grösse dem vierten bis fünften Monat. In ihm befinden sich zwei Fötus männlichen Geschlechts. Die Placenten sind deutlich von einander getrennt, und jeder Fötus hat ein besonderes Chorion. Wir haben es also mit Zwillingen zu thun, die aus zwei Eiern hervorgegangen sind.

Die Länge der Fötus beträgt 9 und $10 \mathrm{~cm}$. In der Entwicklung ihrer Organe bestehen keine makroskopisch sichtbaren Unterschiede. Die Darmöffnung ist von der Geschlechtsöffnung getrennt. Bei beiden Fötus sind die Geschlechtsorgane deutlich 
als männliche $z u$ erkennen. Eine Andeutung des Präputium ist nicht $\mathrm{zu}$ constatiren.

Das rechte Ovarium der Mutter bietet die Zeichen der Oophoritis phlegmonosa. Es ist ödematös und blutreich. Die Oberfläche ist mit stecknadelkopf- bis erbsgrossen, leicht prominenten, bläulichrothen Flecken bedeckt - Hämorrhagieen in die Albuginea. Nirgends die Spur von einem Corpus luteum.

Das linke Ovarium zeigt dieselben Erscheinungen, nur in etwas geringerer Stärke. Es ist $4 \mathrm{~cm}$ lang, $2^{1} / 2 \mathrm{~cm}$ breit, $11 / 2 \mathrm{~cm}$ dick. Es hat die Form einer Wallnuss. Das nach dem Uterus zu liegende Ende ist etwas verdickt, rundlich. Auf dem Durchschnitt erblickt man an diesem medialen Pol einen leicht prominenten Körper. In seiner Mitte befindet sich ein blaurother, rundlicher Kern, der von einem breiten, gelben, gezackten Rand umgeben wird. Um den letzteren zieht sich ein schmaler, weisslicher Ring. Die grösste Länge dieses im Ganzen rundlichen Körpers beträgt $1^{1} / 2 \mathrm{~cm}$, die grösste Breite $1,1 \mathrm{~cm}$. Die geringste Entfernung desselben von dem Rande des Ovariums ist $3 / 4 \mathrm{~mm}$. An dem lateralen, mehr zugespitzten Pol des Ovariums befindet sich ein langgestreckter, halbmondförmiger, leicht erhabener Körper. Die Entfernung der beiden Enden beträgt $1,3 \mathrm{~cm}$, die grösste Breite $0,7 \mathrm{~cm}$. Die geringste Entfernung vom Rande des Ovariums ist $1 \mathrm{~mm}$. Der Längsrichtung dieses Körpers entsprechend zieht in seiner Mitte ein schmaler, weisslicher Streifen, von dem nach den Seiten astartige Ausläufer ausgehen. Zwischen diesen liegt eine gelbe Zone, die etwas breiter als der Mittelstreifen ist. Sie wird wiederum von einer weisslichen Schicht umgeben.

Ich habe durch das in Formalin conservirte und in Alkohol gehärtete Ovarium, von dem die eine Hälfte in Paraffin, die andere in Photoxylin eingebettet wurde, cine sehr grosse Anzahl von Schnitten gemacht. An ihnen habe ich gesehen, dass in allen Ebenen die beiden soeben geschilderten Körper verhältnissmässig dieselben Unterschiede bewahrten. Schon beim Durchführen des Messers auf dem Mikrotom fiel die grössere Härte des kleineren Körpers auf.

Der genaue mikroskopische Befund ist folgender:

Der grössere rundliche Körper ist von einer bindegewebigen Schicht, die mit zahlreichen Rundzellen durchsetzt ist, umgeben. In ihr liegen reirhlich Blutgefässe. Von dieser Schicht gehen nach dem Innern des Körpers schmale, bindegewebige Züge aus, die 
sich baumartig verzweigen. Sie ragen in eine breitere Zone ron grossen, unregelmässig geformten Zellen hinein, welche sich schlecht gefärbt haben und gar keinen oder nur. undeutlichen Kern erkemnen lassen. Zwischen diesen liegen durch ihre bessere Färbung auffallende Bindegewebszellen in feinen Zügen, die von den soeben erwähnten bindegewebigen Strängen ausgehen. Die innere Begrenzung dieser grosszelligen Zone bildet eine schmale bindegewebige Schicht, von der nach der Peripherie zu Ausläufer ausgehen, deren Verzweigungen mit denen von aussen ihnen entgegenkommenden communiciren. Nach der Mitte zu lockert sich das Bindegewebe immer mehr auf. Es liegen zwischen ihm leere Räume, und den grossen Kern des Körpers bildet eine nur wenig gefärbte Masse, die aus feinen Fäden (Fibrin) und zerfallenen rothen Blutkörperchen besteht.

Der zweite, kleinere Körper zeigt ebenfalls eine bindegewebige Umrandung. Die nach innen von ihr gelegene Zone besteht auch aus grossen Zellen und hat ungefähr die gleiche Breite, wie die des anderen Körpers. Nur sind die vom Centrum und der Peripherie in diese Schicht eindringenden Bindegewebsstränge zahlreicher und breiter als die ihnen entsprechenden Gebilde. Der langgezogene, schmale Kern des Körpers wird von einer soliden Bindegewebsmasse gebildet. Fibrin und Reste von Blutkörperchen sind nicht nachweistar. Besonders fällt der Unterschied zwischen dieser schmalen, festen Bindegewebsleiste und dem reichlichen, lockeren Gewebe in der Mitte des grossen Körpers auf.

Ich bemerke noch, dass die beschriebenen zwei Körper unter Berücksichtigung aller Schnitte geschildert sind.

Wir haben es im vorliegenden Fall mit einer Zwillingsschwangerschaft zu thun. Der Umstand, dass zwei deutlich getrennte Placenten vorhanden sind, und dass jeder der beiden Fötus ein eigenes Chorion hat, veranlasst uns zu der Annahme, dass die Zwillinge aus zwei Eiern hervorgegangen sind. Zwischen den beiden Zwillingen hesteht eine Grössendifferenz. Wie ich bereits in der Einleitung erwälınte, ist darauf im Allgemeinen kein Gewicht zu legen. Sie ist keine seltene Erscheinung, und man braucht ihrethalben allein nicht anzunehmen, dass der kleinere Fötus jünger sei, also nacherzeugt sei, als der andere bereits gebildet war. Der sogenannte Kampf ums Dasein im Mutterleib erklärt hinreichend die Ungleichheit in der Grösse.

Die Vermuthung, dass wir aus zwei Eiern hervorgegangene 
Zwillinge vor uns haben, wird durch den Befund in den mütterlichen Eierstöcken bestätigt. In dem linken Ovarium finden wir zwei Corpora lutea. Zwischen diesen besteht nun ein sehr auffallender Unterschied. Sie sind verschieden gross, zeigen verschiedene Form und sind ungleich weit von der Oberfläche des Ovariums entfernt. Besonders wichtig aber ist, dass ihr feinerer Bau sowohl makroskopisch, wie noch mehr bei mikroskopischer Betrachtung wesentliche Unterschiede zeigt. Das grosse Corpus luteum hat ausser der bindegewebigen Umrandung und der grosszelligen (Luteinzellen) Schicht einen ungefähr die Hälfte des ganzen Körpers einnehmenden centralen Kern, bei dem nur die nach aussen gelegenen Partieen von lockerem Bindegewebe gebildet werden, während das Innere von einer aus Fibrin und Resten von Blutkörperchen gebildeten Masse erfüllt ist. Wir haben hier also die Zeichen eines früher erfolgten Blutergusses vor uns.

Ganz anders ist der Befund bei dem kleineren Corpus luteum. Schon die beim Schneiden bemerkbare grössere Härte weist auf das Vorhandensein von mehr Bindegewebe hin. Damentsprechend sehen wir die in die Luteinzellenschicht eindringenden Bindegewebszapfen breiter und zahlreicher als bei dem anderen Corpus luteum. Besonders auffallend ist der Unterschied in der Beschaffenheit der innersten Schicht. In ihr sind keine Spuren von einem Bluterguss zu sehen. Sie besteht aus einer festen Bindegewebsmasse. An die Stelle des Blutergusses ist eine bindegewebige Narbe getreten. Das kleinere Corpus luteum befindet sich also in einem Zustand weiter vorgeschrittener regressiver Metamorphose. Erklärt wird dieser Unterschied in der Entwicklung durch die Annahme, dass der dem kleineren Corpus luteum entsprechende Follikel früher geplatzt ist. Wie gross die Zeitdifferenz gewesen sein mag, ist nicht mit Bestimmtheit zu sagen. Dazu müssten wir eine genaue chronologische Kenntniss der Veränderungen bei der Rückbildung der gelben Körper haben. Jedenfalls können wir vermuthen, dass einige Wochen Altersunterschied die verschiedenartige Beschaffenheit bedingt. Mit dieser Annahme stimmt auch die verschiedene Grösse der Fötus überein. So wenig diese Differenz allein zu bedeuten hat, um so wichtiger ist sie im Verein mit dem Befund an den gelben Körpern.

Ich will nicht bestreiten, dass man die Ungleichheit der Corpora lutea auch anders deuten kann. Man kann eine verschiedene 
Blutzufuhr zu ihnen, also verschiedene Ernährungszustände annehmen, die das schlechter mit Nahrungsmaterial versorgte sich langsamer zurückbilden lassen. Es ist doch aber gesucht, ohne jede Veranlassung eine circumscripte Ernährungsstörung im Eierstock zu vermuthen und dann noch eine andere für den einen Fötus im Uterus anzunehmen. Ferner könnte man einwenden, dass das jüngere Corpus luteum aus einem nach dem Bestehen der Zwillingsschwangersehaft geplatzten Follikel hervorgegangen sei. Wir müssten uns den Vorgang dann so vorstellen, dass zuerst ein Follikel, der zwei Eier enthielt, geplatzt sei. Diese seien befruchtet worden. Dann wäre nach einiger Zeit ein anderer Follikel gesprungen, dessen Corpus luteum unter dem Einfluss der durch die Schwangersehaft bedingten Hyperämie die geschilderte Grösse erreicht babe. Diese Erklärung ist aber jedenfalls sehr gekünstelt.

Viel einfacher ist es, anzunehmen, dass die beiden Corpora lutea aus den beiden geplatzten Follikeln der nachher befruchteten Eier hervorgegangen sind. Die Follikel sind nicht zu gleicher Zeit, sondern einige Wochen nach einander geplatzt, und dementsprechend stehen sowohl die Fötus, wie die Corpora lutea in ihrer Entwicklung auf verschiedenen Stufen. Z Zu dem weiter ausgebildeten Fötus gehört das mehr zurückgebildete Corpus und umgekehrt. Wenn diese Annahme zutrifft, haben wir also einen Fall vor uns, bei dem einige Wochen nach erfolgter Conception noch ein zweites Ei befruchtet wurde, einen Vorgang, den man wohl am besten mit Superfoetatio bezeichnet.

Herrn Geheimrath Professor Dr. Virchow sage ich für die Ueberlassung des Arbeitsplatzes meinen verbindlichsten' Dank.

\section{Erklärung der Abbildungen auf Tafel XI.}

Figur 1. Durchschnitt durch das linke Ovarium in natürlicher Grösse.

Figur 2. Das kleinere Corpus luteum in $\mathbf{1 6}$ facher Vergrösserung.

Figur 3. Das grössere Corpus luteum in 16 facher Vergrösserung. 


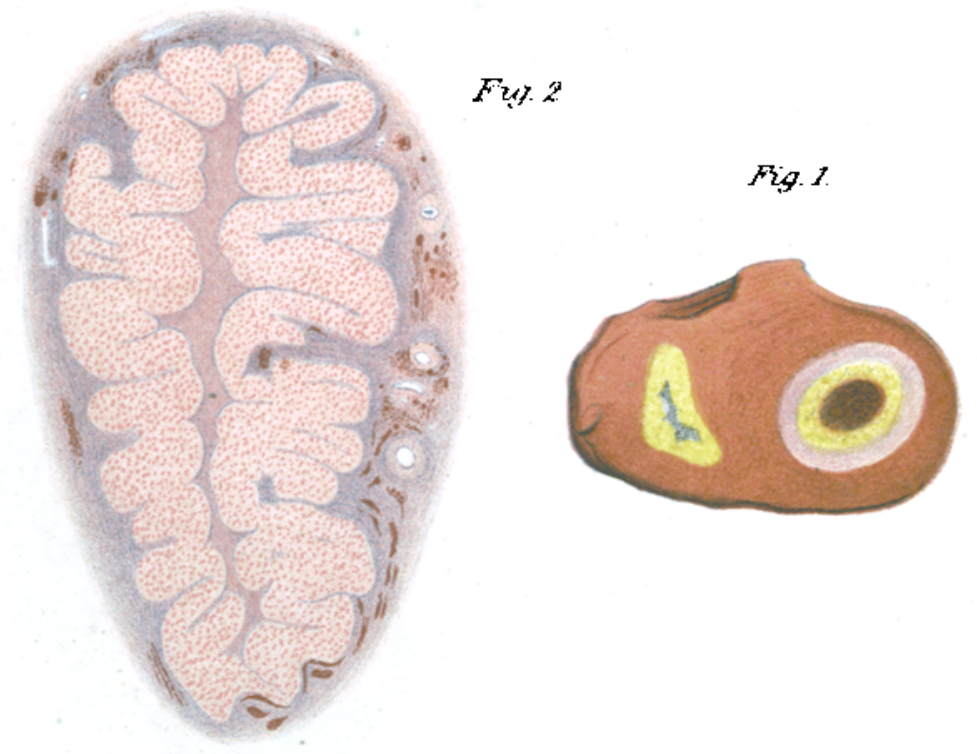

Fig. 3

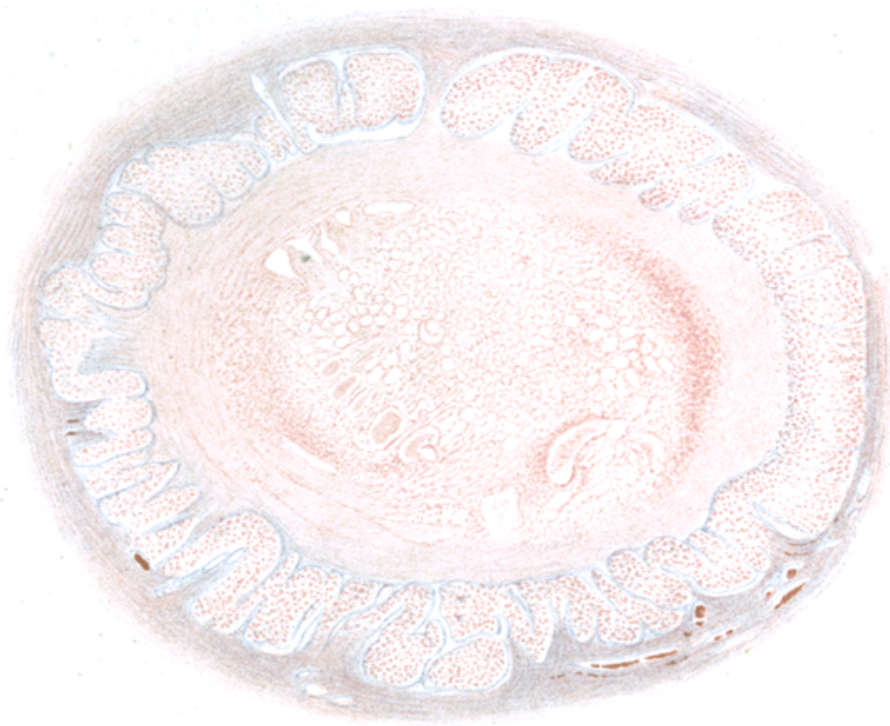

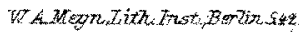

